\title{
Efficacy of Anti-Epileptic Drugs in the Treatment of Tumor and Its Associated Epilepsy: An in vitro Perspective
}

\author{
Taranjeet Kaur ${ }^{\mathrm{a}}$ Shaffi Manchanda ${ }^{\mathrm{a}}$ Vedangana Saini $^{\mathrm{a}}$ \\ Sukhwinder S. Lakhman ${ }^{\text {b }}$ Gurcharan Kaur ${ }^{\text {a }}$ \\ a Department of Biotechnology, Guru Nanak Dev University, Amritsar, India; ${ }^{\text {b}}$ Department of Pharmaceutical, \\ Social and Administrative Sciences, D'Youville College School of Pharmacy, Buffalo, N.Y., USA
}

\section{Key Words}

Tumor associated epilepsy - Cytoprotection .

Differentiation · Plasticity markers

\begin{abstract}
The change in the therapeutic targets from neuron to glia has proved beneficial in the treatment of many psychiatric disorders. The anti-epileptic drugs (AEDs) have been widely prescribed for the treatment of partial and complete seizures, bipolar disorder among others. The current study was carried out to explore the efficacy of some conventional and novel AEDs for the treatment of tumor-associated epilepsy which develops in $29-49 \%$ of the patients diagnosed with brain tumors. We used C6 glioma cell line as model system to study the effect of selected AEDs, viz., gabapentin (GBP), valproic acid (VPA) and topiramate (TPM). Morphometry, cell cycle analysis, apoptosis, expression of different protein markers, viz., GFAP, HSP70 and nuclear factor-kB (NFkB) were studied in AED-treated cultures. The study was further extended to rat hypothalamic primary explant cultures, and cell migration and expression of plasticity markers - neural cell adhesion molecule (NCAM) and polysialylation of NCAM (PSA-NCAM) - were studied in the explants. TPM was observed to show more pronounced increase in apoptosis of
\end{abstract}

glioblastoma cells accompanied by significant downregulation in the expression of HSP70 and NFKB. TPM-treated explants also showed highest process ramification and cellular migration accompanied by intense expression of the plasticity markers as compared to those treated with GBP and VPA. Among the 3 AEDs tested, TPM was observed to show more promising effects on cytoprotection and plasticity of $\mathrm{C} 6 \mathrm{gli}-$ oma cells.

(c) 2016 S. Karger AG, Basel

\section{Introduction}

Over the last 2 decades, a new hypothesis has emerged that defines the dependence of neuronal function and seizure susceptibility on glial cells. Several studies suggest the role of glia as a potential therapeutic target for the treatment of epilepsy and other CNS diseases $[1,2]$. Antiepileptic drugs (AEDs) have been widely prescribed for the treatment of tumor-associated epilepsy. The targets of most current AEDs are neuronal voltage-gated sodium channels and calcium channels, glutamate receptors or

T.K. and S.M. have contributed equally.

\section{KARGER}

E-Mail karger@karger.com

www.karger.com/aon
(C) 2016 S. Karger AG, Basel

0972-7531/16/0231-0033\$39.50/0
Prof Gurcharan Kaur

Department of Biotechnology

Guru Nanak Dev University

Amritsar, Punjab 143005 (India)

E-Mail kgurcharan.neuro@yahoo.com 
GABA neurotransmission [3]. There are a number of adverse side effects associated with the use of AEDs, which often lead to treatment failure [4]. Thus, new AEDs targeting glial cells may enhance efficacy as well as reduce side effects as compared to their neuronal targets.

Topiramate (TPM), a novel AED, has been reported to be neuroprotective and rescues the oligodendrocytes in models of traumatic brain injury [5]. Also, animal model studies suggest that newer AEDs, such as levetiracetam, TPM and zonisamide, may have neuroprotective or antiepileptogenic properties [6]. Valproic acid (VPA) has also been reported to exert neuroprotective effects in vivo in several cellular and animal models of amyotrophic lateral sclerosis disease [7]. Furthermore, VPA has been reported to protect the culture of cerebellar neurons against glutamate-induced neurotoxicity [8].

Another AED, gabapentin (GBP) is prescribed for the cure of pain, alterations of sensation and pruritus associated with skin diseases [9], movement disorders, migraine prophylaxis and cocaine dependence [10]. Recent studies have suggested the neuroprotective role of GBP in hippocampal and cortical neurodegeneration $[11,12]$ and on spinal cord ischemia-reperfusion injury in rabbits [13].

The aim of our study was to test glioprotection and glioplasticity-inducing potential of conventional and new generation AEDs, namely GBP, VPA and TPM. The 3-(4,5-dimethylthiazol-2-yl)-2,5-diphenyltetrazolium bromide (MTT) assay was performed to initially select the appropriate dose of these AEDs. Later, cell cycle analysis was carried out to determine the stage at which selected doses of these drugs arrest the cell cycle. Additionally, toxicity and/or induction of apoptosis or necrosis effect of these AEDs were also assessed. Morphometric analysis was done to demonstrate the neurite outgrowth potential of these AEDs. And further effect of these AEDs on primary cultures, migration and process outgrowth was assessed using hypothalamic primary explant cultures. Together our findings provide insights on the potential role of GBP, VPA and TPM in glioprotection, and neuronal and glial plasticity that may contribute to the roles of these AEDs as novel therapeutics.

\section{Methods}

\section{Cell Culture and Treatments}

C6 glioma cell line was obtained from National Centre for Cell Science, Pune, India. Cells were cultured in $75-\mathrm{cm}^{2}$ vented flasks using Dulbecco's Modified Eagle Medium (Sigma Aldrich, USA), which was supplemented with $1 \times$ PSN (Life Technologies,
USA) and 10\% fetal bovine serum (Biological Industries, Israel). Cultures were grown in an incubator at $37^{\circ} \mathrm{C}, 5 \% \mathrm{CO}_{2}$ and $95 \%$ relative humidity. Undifferentiated cells were subcultured by trypsinization $(0.01 \%$ PBS) when they reached $70-80 \%$ confluency. Cells were seeded in the split ratio of 1:2. The media was changed every other day. The treatment with AEDs was given $2 \mathrm{~h}$ after seeding for $72 \mathrm{~h}$ in multi-well plates. After treatment, the cells were harvested for viability assays, phase contrast microscopy, immunofluorescence studies and Western blotting. The control group was given a media change alone. The cultures were maintained at low passage numbers and were free of mycoplasma contamination.

\section{Cell Viability Assay}

The cell viability was assessed by MTT assay [14]. C6 cells at $30-40 \%$ confluency were treated with $1-100 \mu \mathrm{M}$ concentrations of AEDs (GBP, VPA and TPM; Sun Pharmaceutical Ind., Ltd., India) and incubated for $72 \mathrm{~h}$. The cell viability was estimated by measuring the absorbance of purple colored formazan crystals formed by the activity of mitochondrial dehydrogenases.

\section{Cell Morphology Studies}

Morphological changes in C6 glioma cells treated with AEDs were studied by phase contrast microscopy. These experiments were performed in 24 well plates containing cover slips seeded with 10,000 cells $/ \mathrm{ml}$. The phase contrast images of cultures were taken using Phase Contrast Inverted Microscope (Nikon TE2000).

\section{Morphometry}

The effect of AEDs on the cell differentiation was investigated. To achieve that, C6 cells were studied for number and length of process outgrowths. This study was done as described previously in our laboratory [15]. Briefly, C6 cells were seeded at a density of 5,000 cells $/ \mathrm{ml}$ in 48 well plates. After incubation with the AEDs, cells were fixed with $25 \%$ glutaraldehyde solution (final concentration in the medium was $2.5 \%$ ) for 90 min followed by 3 washings with PBS at room temperature. The cells were then stained in a solution containing $1 \%$ toluidine blue and $1 \%$ methylene blue in $1 \%$ borax (sodium tetraborate) for $1 \mathrm{~h}$ at room temperature. The staining solution was then removed and cells were washed with water and air-dried overnight at room temperature. Bright field images were taken using inverted microscope (Nikon TE2000). Length and number of outgrowths were measured using Image Pro Plus software version 4.5.1, and the analysis was done using Microsoft Excel and Sigma Stat for windows (version 3.5).

\section{Annexin V-FITC (Apoptosis) Assay}

To further explore whether AEDs cause apoptosis and necrotic cell death, cell suspension was harvested from cells cultured and treated in $90-\mathrm{mm}$ Petri dishes. Cells from 4 Petri dishes were pooled together. Cells were stained with Annexin V conjugated with FITC and PI using Annexin V-FITC Apoptosis Detection Kit (Miltenyi Biotech, San Diego, Calif., USA), according to the manufacturer's instructions. Briefly, the cells from the control and treated groups were obtained after centrifugation at 5,000 rpm. Cells were resuspended in $1 \mathrm{ml}$ of $1 \times$ binding buffer, and $10 \mu \mathrm{l} \mathrm{An-}$ nexin V-FITC was added and incubated for $15 \mathrm{~min}$ in a darkroom. The pellet was obtained by centrifugation at 5,000 rpm and resuspended in $500 \mu \mathrm{l}$ of $1 \times$ binding buffer and $5 \mu$ lof propidium iodide solution was added immediately prior to analysis by BD Accuri C6 
flow cytometer (BD Biosciences Immunocytometry Systems, San Jose, Calif., USA). Cycloheximide $(50 \mu \mathrm{g} / \mathrm{ml})$ was used as a positive control.

\section{Cell Cycle Analysis}

To elucidate whether AEDs modulate signaling pathways associated with cell cycle, cells were grown and treated in $90-\mathrm{mm}$ Petri dishes. Cells from 4 Petri dishes were pooled together. After $72 \mathrm{~h}$ of treatment, cells were trypsinized and collected along with the floating cells. The pellet was obtained by centrifugation and was resuspended in $1 \mathrm{ml}$ PBS (ice cold). Fixation of cells was done in ice-cold $70 \%$ ethanol. Staining of cells was done with propidium iodide. Cell sorting was done using BD Accuri C6 flow cytometer. Analysis was done using CFlow Plus software (BD Biosciences).

\section{Explant Cultures from Neonatal Rat Brain and AEDs \\ Treatment}

The explant cultures of neonatal rat brain from 2-day-old pups were performed in part as described by Boss et al. [16]. Animal care and procedures were followed in accordance with the guidelines of the Animal Ethical Committee, Guru Nanak Dev University, Amritsar, India. Briefly, rat pups were sacrificed by decapitation. Brain was removed from the cranium and kept in sterile ice-cold Solution I ( $1 \times \mathrm{KRB}, 1.2 \mathrm{mM} \mathrm{MgSO}_{4}, 3 \mathrm{mg} / \mathrm{ml} \mathrm{BSA}$; KRB: $120 \mathrm{mM} \mathrm{NaCl}$, $5 \mathrm{mM} \mathrm{KCl}, 1.2 \mathrm{mM} \mathrm{KH}_{2} \mathrm{PO}_{4}, 25 \mathrm{mM} \mathrm{NaHCO}_{3}, 14 \mathrm{mM}$ glucose, $0.015 \%$ phenol red). Hypothalamus was separated from the brain, and meninges around the tissue were removed by gentle rolling on sterile filter paper sheet. The separated region was kept in $3 \mathrm{ml}$ of Solution I. The tissue was minced into $0.2 \mathrm{~mm}$ pieces with a sterile scalpel by moving in 2 orthogonal directions. The minced tissue was kept on poly-L-lysine coated cover slips in a multi-well plate and covered with $50 \mu \mathrm{l}$ of Neurobasal medium (Life Technologies, USA). It was incubated at $37^{\circ} \mathrm{C}$ in a humidified $5 \% \mathrm{CO}_{2}$ in a $\mathrm{CO}_{2}$ incubator. After $2 \mathrm{~h}$, the medium was replenished and explants treated with AEDs in different wells for $72 \mathrm{~h}$.

\section{Immunofluorescence Staining}

All cells/explants including the control as well as the treated ones were washed with $1 \times$ PBS thrice for 5 min each and then fixed with $4 \%$ paraformaldehyde. Cells/explants were given a single wash with $1 \times$ PBS and permeabilization was carried out with $0.3 \%$ Triton X-100 in PBS (PBST) for 15 min followed by blocking with $2 \%$ BSA (prepared in PBS) for $30 \mathrm{~min}$ at room temperature. Cells/explants were incubated with the respective primary antibody (rabbit anti-GFAP (1:300); mouse anti-HSP70 (1:300); rabbit anti-GAD (1:200); mouse anti-NFkB (1:250); Sigma Aldrich, USA) prepared in $2 \% \mathrm{BSA}$ at $4^{\circ} \mathrm{C}$ overnight in a humid chamber. The cells/explants were then washed with $0.1 \%$ PBST thrice and incubated with respective secondary antibody (Alexa Fluor 488 goat anti-mouse IgG (1:500) for NFkB; Alexa Fluor 546 goat antimouse IgG (1:500) for HSP70; Alexa Fluor 488 goat anti-rabbit IgG for GFAP (1:500) and GAD (1:200)) (Life Technologies, USA) for $2 \mathrm{~h}$ in a humid dark chamber at room temperature. Then the cells/explants were incubated with DAPI $(1: 10,000)$ (Sigma Aldrich, USA) for $10 \mathrm{~min}$ in a humid dark chamber at room temperature. Finally, the cells/explants were washed thrice with $0.1 \%$ PBST followed by $1 \times$ PBS and cover slips containing cells/explants were then mounted on glass slides using Fluoromount (Sigma Aldrich, USA). Images were captured using A1R confocal microscope (Nikon).

Efficacy of AEDs in the Treatment of Tumor and Its Associated Epilepsy
For dual immunostaining, explants without permeabilization were incubated with a mixture of primary antibodies mouse antipolysialylation of neural cell adhesion molecule (PSA-NCAM, 1: 250; Merck Millipore, USA) and mouse anti-NCAM (1:250; Sigma Aldrich, USA) prepared together in $2 \% \mathrm{BSA}$ at $4^{\circ} \mathrm{C}$ overnight. The cells were then washed with $1 \times$ PBS and incubated with secondary antibodies (Alexa Fluor 488 goat anti-mouse IgM and Alexa Fluor 546 goat anti-mouse IgG 546 (1:250 each); Life Technologies, USA) in $2 \%$ BSA for $2 \mathrm{~h}$ in humid dark chamber at room temperature and counterstained with DAPI (nuclear stain).

\section{Protein Assay and Western Blotting}

Cells were grown and treated in $90-\mathrm{mm}$ Petri dishes and were harvested with $1 \mathrm{ml}$ PBS-EDTA (0.1 mM EDTA in $1 \times$ PBS) after scraping. The cell pellet was homogenized in chilled homogenizing buffer (RIPA) followed by centrifugation for $10 \mathrm{~min}$ at 5,000 rpm. Supernatant was collected and protein content was determined by Bradford assay. Samples were prepared after equilibrating the protein in all groups. The samples were then mixed with $6 \times$ sample buffer (0.25 M Tris- $\mathrm{HCl}$ (pH 6.8), 20\% glycerol, $4 \%$ sodium dodecyl sulfate (SDS), $10 \% \beta$-mercaptoethanol and $1 \mathrm{mg}$ bromophenol blue) and stored at $-20^{\circ} \mathrm{C}$. Cell lysates $(30 \mu \mathrm{g})$ were resolved on $10 \%$ SDS-PAGE gels and transferred onto a PVDF membrane (Hybond-P; GE Healthcare, USA) using the semidry TE70 PWR system (Amersham Biosciences, USA). Subsequently, the membrane was blocked (5\% skimmed milk in $0.1 \%$ TBST) and immediately incubated with respective primary antibody rabbit antiGFAP (1:2,000); mouse anti-HSP70 (1:5,000); mouse anti-NFkB $(1: 2,000)$ (Sigma Aldrich, USA) at $4{ }^{\circ} \mathrm{C}$ overnight, followed by the respective secondary antibody (goat anti-rabbit for GFAP and goat anti-mouse for HSP70 and NFKB) conjugated with horse radish peroxidase (HRP) (1:5,000; Merck Millipore, USA) for $2 \mathrm{~h}$ at room temperature. The membranes were also probed with anti- $\alpha$ tubulin antibody (1:5,000; Sigma Aldrich, USA) as an internal control for protein loading. Immunoreactive bands on membrane were visualized using ECL Prime Western Blotting Detection Reagent (GE Healthcare, USA) according to the manufacturer's instructions and the antibody-labeling intensity (relative optical density) was quantified using Image Quant LAS 4,000 (GE Healthcare, USA).

\section{Statistical Analysis}

Values are expressed as mean \pm SEM from at least 3 independent experiments. The Sigma Stat for Windows (version 3.5) was adopted to analyze the results by one-way analysis of variance in order to determine significance of the means. Values of $\mathrm{p}<0.05$ were considered as statistically significant.

\section{Results}

\section{Cell Viability Assays}

To determine that the selected concentration of AEDs is not cytotoxic and has the potential to induce glioprotection, cell viability of these compounds was tested using MTT assay. Cells were treated with different concentrations of GBP $(1-100 \mu \mathrm{M})$, VPA $(1-100 \mu \mathrm{M})$ and TPM (1- 

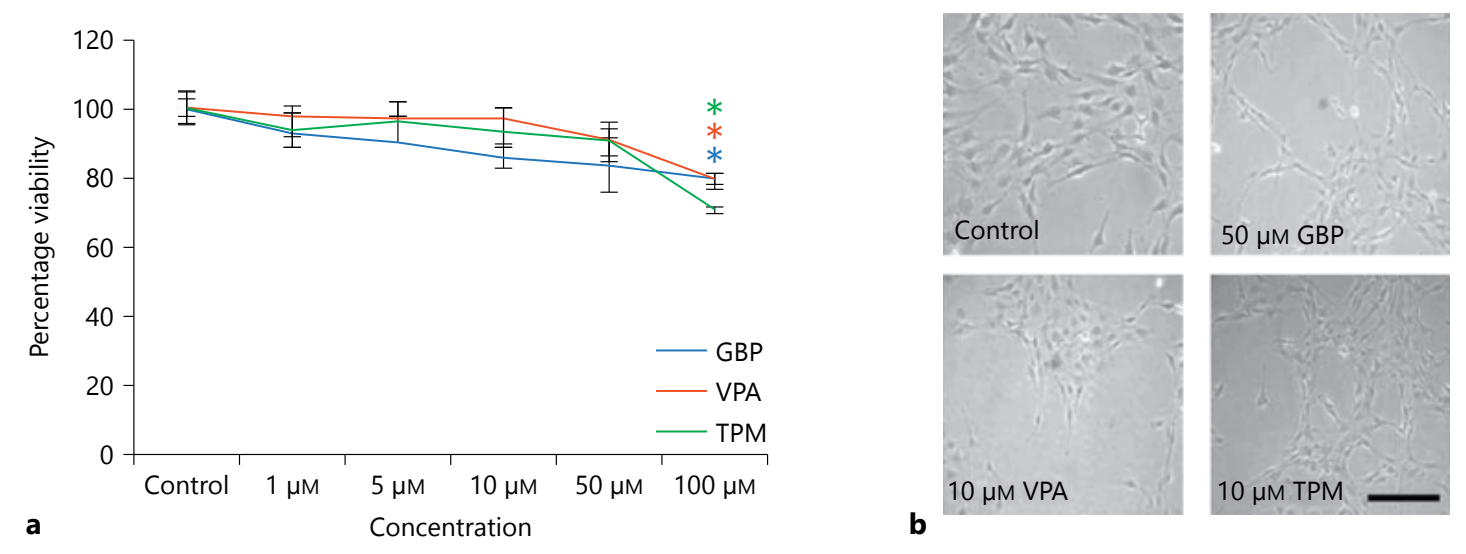

Fig. 1. Cell viability assay. a Percentage viability of C6 cells after $72 \mathrm{~h}$ of treatment with different concentrations of GBP, VPA and TPM. * Represents statistically significant difference $(\mathrm{p}<0.05)$ between control and drug-treated groups. The experiment was per-

$100 \mu \mathrm{M})$ for $72 \mathrm{~h}$ (fig. 1a). Significant change in cell morphology, along with considerable rounding in cells, was observed in GBP-treated cells at a concentration of 100 $\mu \mathrm{M}$ and in VPA- and TPM-treated cells from $50 \mu \mathrm{M}$ onward (data not shown). Significant downregulation in cell viability was observed at $100 \mu \mathrm{M}$ concentration in all the treatment groups. For further studies, the sub-toxic concentrations of AEDs used were 50, 10 and $10 \mu \mathrm{M}$ for GBP, VPA and TPM, respectively.

\section{AEDs Induce Differentiation-Like Morphology in Glioblastoma Cells}

To elucidate morphological changes in C6 cells after treatment with AEDs, phase contrast images of treated and control cultures were taken (fig. 1b). Treatment of C6 cells with GBP, VPA and TPM resulted in their differentiation showing longer processes accompanied by the shortening of cell body as compared to untreated control cells. Further morphometric analysis was done to compare the number and length of the processes in control and treated cells. After $72 \mathrm{~h}$ of incubation with the AEDs, the length of processes in GBP-, VPA- and TPM-treated cells showed a significant increase ( $p<0.05$; fig. $2 a$ ), though the number of processes in treated cells and control cells were almost similar (fig. 2b).

\section{Annexin V-FITC Assay and Cell Cycle Analysis}

To further determine whether the selected doses of AEDs cause apoptotic effect, Annexin V-FITC assay was performed (fig. 3a, b). GBP-treated cells showed 82.8\% formed in triplicates. $\mathbf{b}$ Phase contrast images of C6 control, $50 \mu \mathrm{M}$ GBP, $10 \mu \mathrm{M}$ VPA and $10 \mu \mathrm{M}$ TPM-treated cells. Images were captured using Nikon TE2000 Phase Contrast Microscope. Scale bar represents the length of $200 \mu \mathrm{M}$. viable cell population, which was almost comparable to control untreated cells (80.8\%), and the number of cells in late apoptosis decreased to $4.1 \%$ after treatment with GBP. With $10 \mu \mathrm{M}$ of TPM, viable cell population was reduced to $74.2 \%$, early apoptotic cells were $17.6 \%$ and late apoptotic cells were $7.1 \%$ as compared to $80.8,13.0$ and $5.3 \%$, respectively, in case of control cultures. No significant changes were observed in the percentage of viable and apoptotic cells in VPA-treated group as compared to the control group, whereas cycloheximide used as positive control reduced the percentage of live cells and increased the number of apoptotic and necrotic cells (fig. 3a, b). The percentage of necrotic cells in all treatment groups was almost negligible. Additionally, cell cycle analysis (fig. 3c, d) showed the majority of the cells in G0/G1 phase in control group. The number of cells in G0/G1 phase increased with all the AEDs, and VPA-treated cells showed maximum cell number in G0/G1 phase. These data again suggest that AEDs may tend to induce differentiation in C6 glioma cells.

\section{Immunofluorescent Staining and Western Blotting}

In the current study, GFAP expression in GBP-, VPAand TPM-treated cells was downregulated with respect to control cells (fig. 4a-d). Similar trend was observed with Western blotting data but the change was not statistically significant (fig. $4 \mathrm{~m}, \mathrm{n}$ ). Furthermore, the effects of selected AEDs on cellular stress response, the expression of marker proteins HSP70 and NFKB were evaluated. The expression of $\mathrm{NF \kappa B}$ was upregulated upon treatment of 


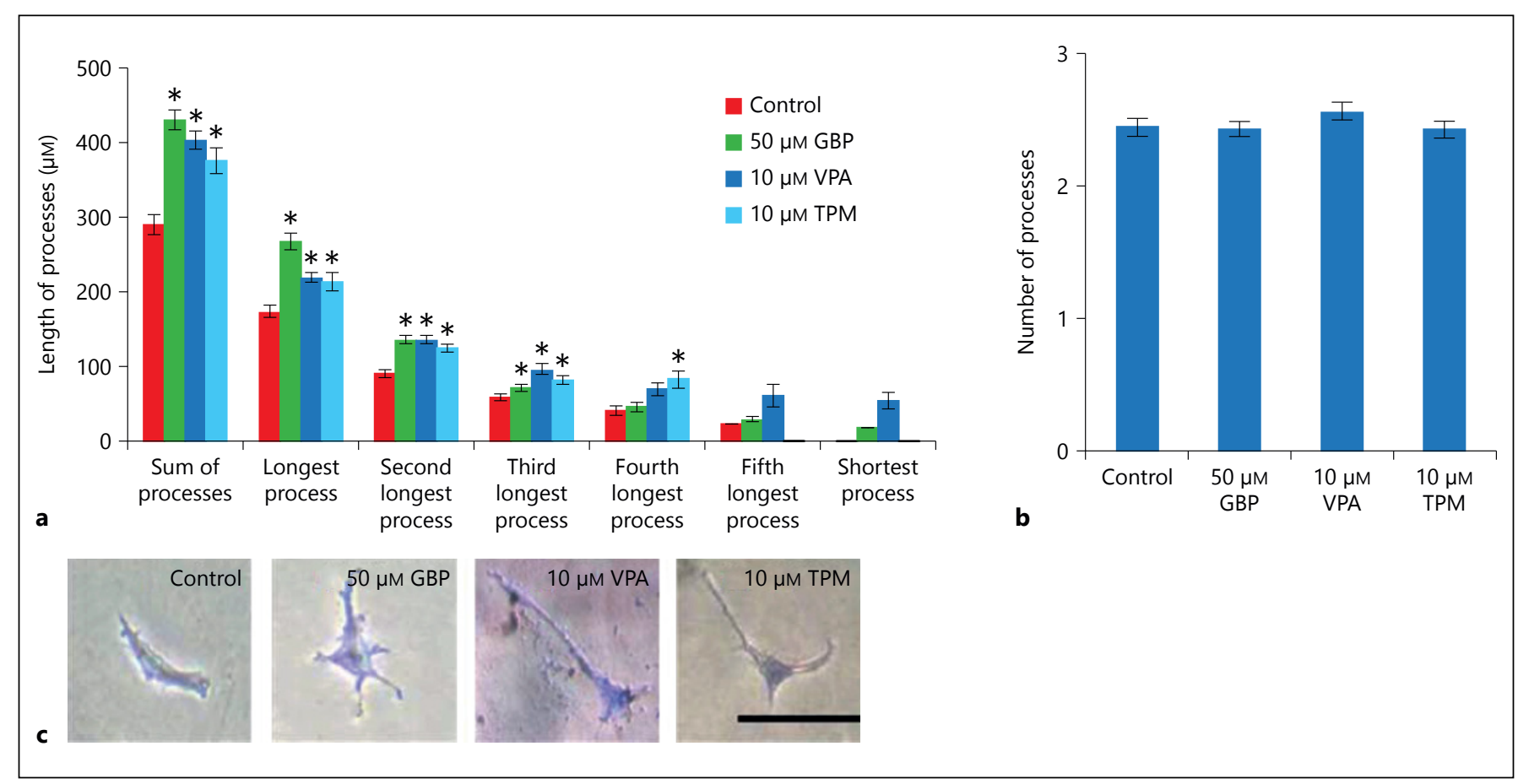

Fig. 2. Morphometry study. The histograms represent the changes in length of processes (a) and number of processes (b) in C6 cells treated with $50 \mu \mathrm{M}$ GBP, $10 \mu \mathrm{M}$ VPA and $10 \mu \mathrm{M}$ TPM with respect to control cells. The data are shown as the average of hundred cells.
* Represents statistically significant difference $(\mathrm{p}<0.05)$ between control and drug-treated groups. c Representative images of cells in each group. Scale bar represents the length of $50 \mu \mathrm{M}$. cells with GBP and VPA, whereas it was significantly $(\mathrm{p}<$ 0.05 ) downregulated upon treatment with TPM as is shown in immunostaining (fig. 4e-h) and Western blotting data (fig. $4 \mathrm{~m}, \mathrm{n}$ ). Cells treated with GBP and VPA showed no change in expression of HSP70, whereas TPM-treated cells showed $16.4 \%$ decrease in HSP70 expression though not at significant level on both immunostaining (fig. $4 \mathrm{i}-\mathrm{l}$ ) and immunoblotting (fig. $4 \mathrm{~m}, \mathrm{n}$ ).

\section{Cellular Migration and Process Outgrowth from}

Hypothalamic Explant Cultures

The cellular migration and process outgrowth was observed in hypothalamic primary explant cultures of 2-day-old pups upon treatment with selected AEDs (fig. 5). TPM-treated explants showed maximum migratory potential as compared to other drugs. Both the migration of cell bodies and process outgrowth was observed in VPA- and TPM-treated explant cultures particularly in the cells at the periphery of explant, whereas GBP treatment did not show any cellular migration or process outgrowth. Since the selected AEDs are known to act through GABAergic pathway and GAD is the key enzyme in the synthesis of GABA, GAD expression was also studied in the hypothalamic explant cultures, which was enhanced (fig. 5a-d) both at the core and periphery of explants with AEDs treatment. To further analyze the plasticity inducing potential of the selected AEDs, the expression of plasticity markers such as PSA-NCAM and NCAM was also studied. Upregulation in the expression of PSA-NCAM and NCAM was observed in the explants treated with GBP, VPA and TPM as compared to control, and TPM-treated cultures showed more pronounced effects (fig. $5 e-p$ ).

\section{Discussion}

In the current study, C6 glioma cell line was used as a model system to study the in vitro effects of the selected AEDs, viz., GBP, VPA and TPM on glioprotection. Our results showed that the moderate doses of these AEDs were not cytotoxic. GBP treatment resulted in dose-dependent decline in cell viability with significant decrease at $100 \mu \mathrm{M}$ concentration; so $50 \mu \mathrm{M}$ concentration of GBP was chosen for further studies. In VPA- and TPM-treated cells, the viability was good up to $10 \mu \mathrm{M}$ concentration, 


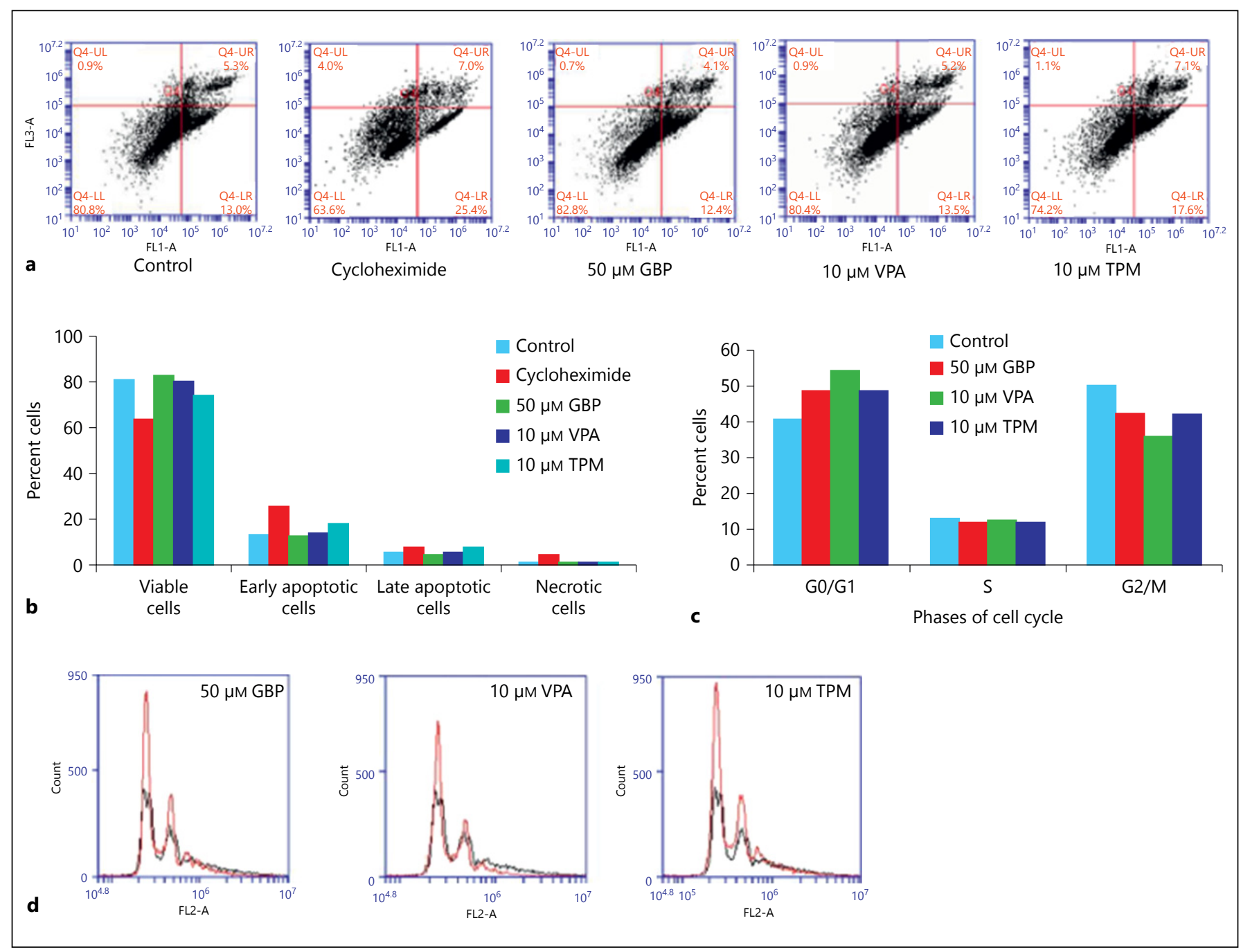

Fig. 3. Flow cytometric examination of apoptosis and cell cycle. a Flow cytometric examination of apoptosis, necrosis and cell viability - the Annexin V-FITC assay of C6 cells after treatment with $50 \mu \mathrm{g} / \mathrm{ml}$ cycloheximide (positive control), $50 \mu \mathrm{M} \mathrm{GBP}, 10 \mu \mathrm{M} \mathrm{VPA}$ and $10 \mu \mathrm{M}$ TPM. Four quadrants of plots depict the following: lower left - viable cells (Annexin V-, PI-), lower right - early apoptotic cells (Annexin V+, PI-), upper right - late apoptotic cells (Annexin $\mathrm{V}+, \mathrm{PI}+$ ), upper left - necrotic cells (Annexin V-, PI+).

which started to decline from $50 \mu \mathrm{M}$ onward and was significantly decreased at $100 \mu \mathrm{M}$. Further experiments with VPA and TPM were performed with $10 \mu \mathrm{M}$ dose. The phase contrast images and morphometry data showed C6 cells with elongated processes in the AED-treated cells as compared to control cultures. The maximum elongation of processes was observed in GBP-treated cultures, and the treatment using these AEDs only caused increase in the length of the processes with no change in the number of processes. The data suggest that these b The histogram represents percentage distribution of cells in different quadrants in control and treated groups. c The histogram represents percentage distribution of cells in different phases of cell cycle in control and drug-treated groups. $\mathbf{d}$ Plots representing cell cycle analysis of $\mathrm{C} 6$ cells in control and treated groups. Black line represents control and red line represents respective treatment group.

AEDs induce differentiation like morphology in C6 glioma cells.

As tumor cells are resistant to apoptosis, the apoptosis inducing potential of these drugs might be an additional effect of these AEDs. The number of apoptotic cells was found to have increased (as supported by Annexin VFITC assay) in TPM-treated cells as compared to the control cells, which is suggestive of its inhibitory role in tumor progression. TPM has been shown to play inhibitory role in tumor progression in mice-bearing Lewis lung 


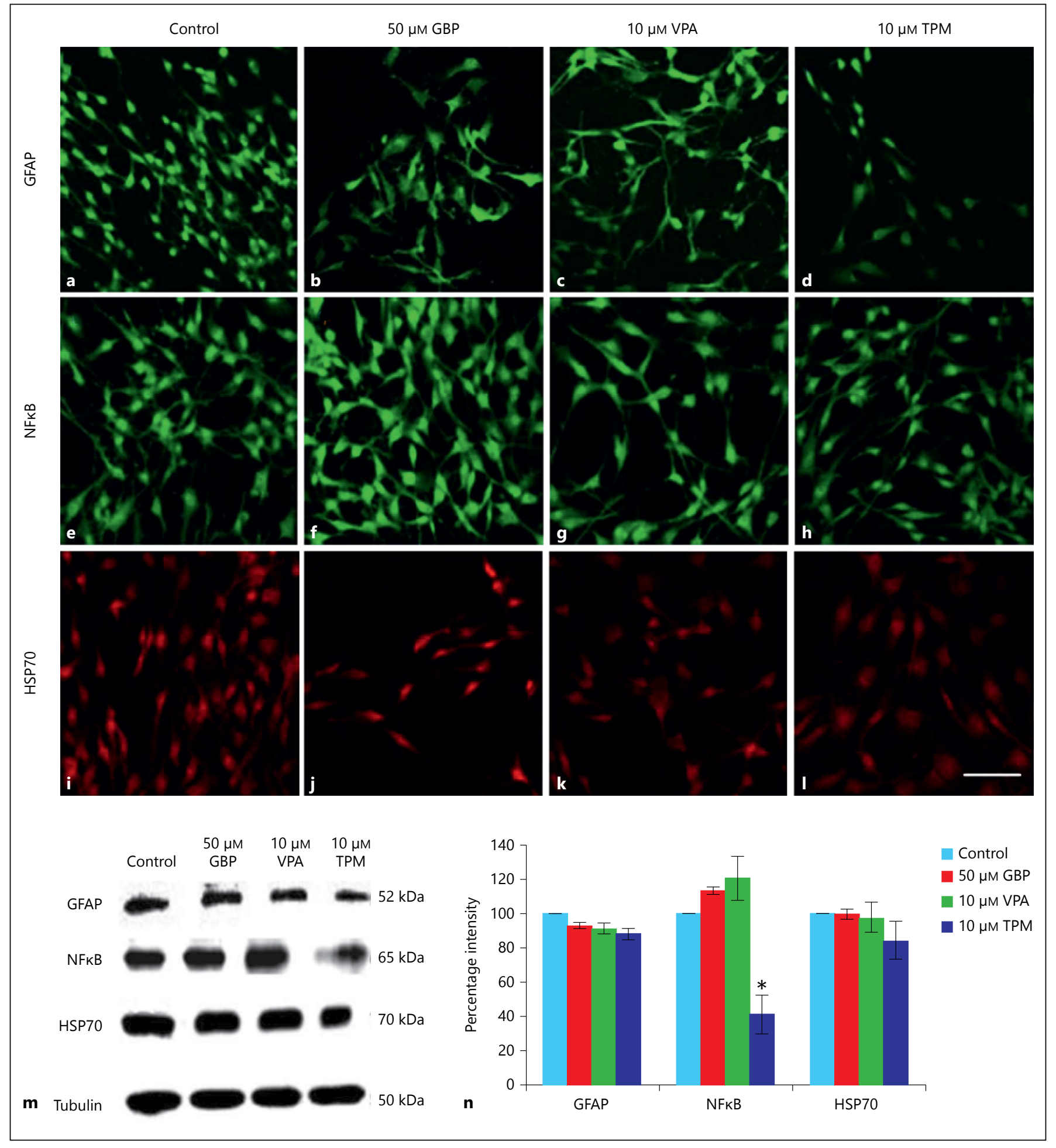

Fig. 4. Immunofluorescent and Western blot analysis. Immunofluorescent staining of GFAP (a-d), NFKB (e-h) and HSP70 (i-I) in control, $50 \mu \mathrm{M}$ GBP, $10 \mu \mathrm{M}$ VPA and $10 \mu \mathrm{M}$ TPM-treated C6 cells. Images were captured using Nikon A1R confocal microscope. Scale bar represents the length of $50 \mu \mathrm{M}$. $\mathbf{m}$ Representative blots for GFAP, NFkB and HSP70 in control and drug-treated C6 cells. $\mathbf{n}$ Quantitative densitometric analysis for markers is shown in histogram. Values are expressed as mean \pm SEM from 3 independent experiments. * Represents statistically significant $(\mathrm{p}<$ 0.05 ) difference in the expression of markers in treated cells with respect to control cells.
Efficacy of AEDs in the Treatment of Tumor and Its Associated Epilepsy
Ann Neurosci 2016;23:33-43 DOI: $10.1159 / 000443554$ 


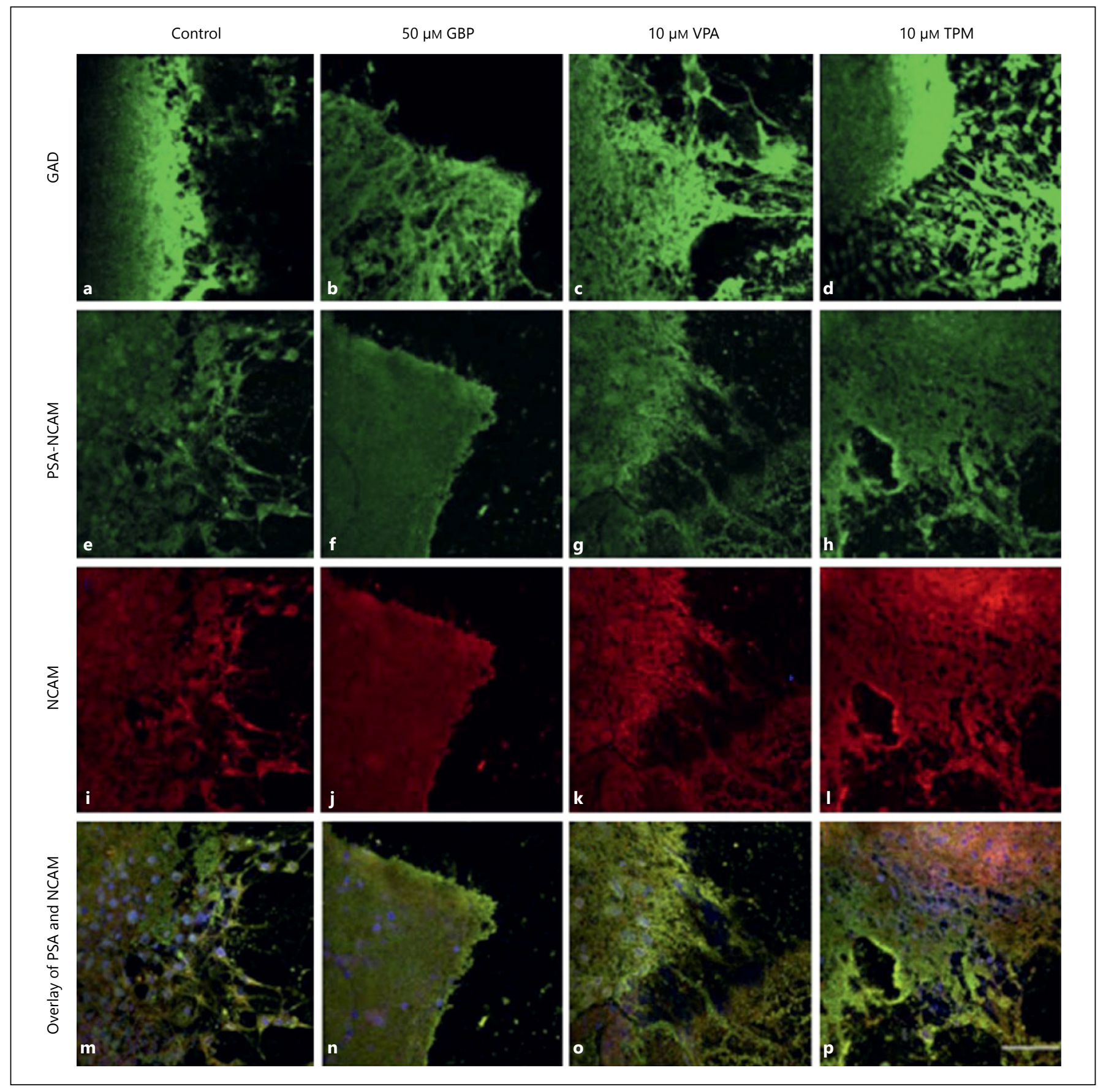

Fig. 5. Primary explant cultures study. Hypothalamic explants from 2-day-old pups stained for GAD (a-d), PSA-NCAM (e-h) and NCAM (i-I). Overlay images $(\mathbf{m}-\mathbf{p})$ show the co-localization of PSA-NCAM and NCAM. Images were captured using Nikon A1R confocal microscope. Nuclei were stained by DAPI. Scale bar represents the length of $50 \mu \mathrm{M}$.

that GBP and VPA are not acting via apoptosis pathway for tumor suppression. Furthermore, the cell cycle analysis showed the majority of the AED-treated cells in G0/ G1 phase with maximum number of cells arrested in G0/ G1 phase seen in VPA-treated cells. The arrest in G0/G1 carcinoma, and this feature has been attributed to the inhibition of angiogenesis by downregulation of osteopontin, VEGF and carbonic anhydrase II $[17,18]$. However, no significant change in number of apoptotic cells was observed in GBP- and VPA-treated cells, which suggests 
phase upon AED treatment is again suggestive of induction of differentiation like phenotype in C6 cells, which subsequently leads to apoptosis as evident from the Annexin V-FITC assay. The regulation of cell cycle and apoptosis pathways play an important role in the growth and development of cancer. Targeting the cell cycle has recently emerged as an attractive approach for the treatment of various cancer types. Anti-cancer drugs currently in clinical use such as paclitaxel [19] and bendamustine [20] have been shown to arrest specific cell cycle phase and also induce apoptosis of target cancer cells. VPA, which is in clinical development phase, has also been shown to selectively arrest cell cycle in G1 phase [21].

Glial cells show reactive gliosis as a generic response to brain damage [22]. The hallmark feature of reactive gliosis is hypertrophy of cellular processes of astrocytes accompanied by upregulation of intermediate filament proteins, including GFAP, which has been reported in CNS trauma, hypoxia, around growing tumors and in many neurodegenerative conditions [23]. In the current study, downregulation in the expression of GFAP upon treatment with selected doses of AEDs in C6 cells may suggest their potential to prevent reactive gliosis. Maximum downregulation in GFAP expression was observed in TPM-treated cell cultures. Thus, these drugs may pose an overall protective effect in tumor-associated epilepsy in patients suffering from glioblastoma, TPM being the most promising drug among the three. GBP has been reported to induce neuroprotection in pilocarpine-induced status epilepticus rats by reducing reactive gliosis as is evident from GFAP downregulation [24]. The expression pattern of many molecular chaperones like heat shock proteins expressed in mitochondria, such as HSP70 have been found to be closely linked to cell proliferation stages in mammals $[25,26]$. Various reports have suggested highly upregulated expression of HSP70 in tumor cell lines; however, in normal cell lines, its expression is only stress inducible [27]. The downregulation of HSP70 expression in tumor cells has been reported to commit them to apoptosis [28]. Downregulation of HSP70 expression in C6 cells after treatment with GBP, VPA and TPM supports the apoptosis data of the current study. Highest percentage of downregulation in HSP70 expression in TPMtreated cultures correlates well with the highest number of early and late apoptotic cells observed in this treatment group.

Expression of $\mathrm{NF} \kappa \mathrm{B}$ was found to be upregulated in GBP- and VPA-treated cells but these changes were not statistically significant. NFKB plays diverse roles ranging from inhibition of apoptosis, induction of differentiation

Efficacy of AEDs in the Treatment of

Tumor and Its Associated Epilepsy to induction of cell survival pathways [29]. Hence, it may be suggested that these drugs induce pathways which help in maintaining homeostasis and stabilizing cellular machinery. In addition, VPA is known to be a potent neuroprotective agent against LPS-induced neurotoxicity, by decreasing levels of proinflammatory factors released from activated microglia [30]. VPA-induced protection has also been reported by Penas et al. [31] after spinal cord injury. NFKB is an anti-apoptotic protein, and a significant $(\mathrm{p}<0.05)$ downregulation in NFKB expression upon treatment with TPM may suggest that the apoptosis induction by TPM may be facilitated by downregulation of NFKB pathway.

Various animal and human studies have reported GABAergic dysfunction in mood disorders and bipolar patients [32]. GAD is the key enzyme responsible for the synthesis of GABA. Our results of hypothalamic explant cultures from 2-day-old pups showed enhanced expression of GAD after treatment with VPA and TPM, with more promising effects in TPM-treated group. It is supported by the potential mechanism of action of these AEDs through GABAergic upregulation as is evident from increased GAD expression in the explant cultures treated with these drugs. It has been proposed that VPA might compensate for a potential GABAergic deficiency through the enhancement of GABA brain concentration [33].

Hypothalamic explant cultures (with normal phenotype) showed intense PSA-NCAM and NCAM immunostaining, the markers of neurite outgrowth, synaptic plasticity and neuronal migration. NCAM is a developmentally regulated protein that has been implicated in various cellular processes, such as cell-cell adhesion, cell migration and synaptic plasticity [34]. Enhanced cellular migration as well as expression of both PSA-NCAM and NCAM, particularly in the cells at the periphery of explant in VPA- and TPM-treated cultures, supports the neuronal-glial plasticity-inducing potential of these drugs. Process outgrowth of migrating cells may suggest that these AEDs promote neurite outgrowth as well as enhance neuronal and glial plasticity. Previous studies have reported that many anticancer compounds inhibit the growth of cancerous cells without arresting the growth of normal cells $[35,36]$. Another study reported that VPA administration enhanced polysialylated NCAM paralleled by reduced invasiveness of tumor cells in various neuroblastoma cell lines in a time-dependent manner [37]. Cellular outgrowth and process ramification were predominantly increased in the periphery of VPA- and TPM-treated explant cultures, with TPM showing more 
pronounced effects. Expression of PSA on NCAM is associated with cellular migration [38]. PSA on the extracellular domain of NCAM reduces cell adhesion by acting as a spacer, allowing dynamic changes in the membrane contacts making extension or outgrowth easier [39]. The current study with AEDs may suggest their potential role in promoting process outgrowth as well as neuronal and glial plasticity through upregulation of plasticity marker proteins. Several previous studies from our lab have reported the potential role of PSA-NCAM in neuronal-glial interactions in adult hypothalamus and neuroendocrine plasticity $[39,40]$.

The people suffering from glioblastoma who have been reported to have epileptic seizure attacks are 29$49 \%$ [41-43]. Our current data suggest that among the 3 AEDs studied, TPM may be the most promising drug for the treatment of tumor and its associated epilepsy by directing the glioma cells toward differentiation and then inducing apoptosis, keeping the surrounding normal cells in cytoprotective environment. Also, these AEDs promote neurogenesis in primary hypothalamic explant cultures that supports the glioprotective and neuronal plasticity inducing potential of these AEDs in normal cells. Since these AEDs are being prescribed for a wide range of clinical conditions such as treatment of neuropathic pain and post-traumatic stress disorder, the current data may add to the unexplored potential of these drugs, which may contribute to the field of glioscience.

\section{Acknowledgments}

T.K. and S.M. are thankful to University Grants Commission (UGC) for fellowship grant under Centre with Potential for Excellence in Particular Area (CPEPA) and University with Potential for Excellence (UPE) schemes during the entire course of study. V.S. is thankful to the CSIR for fellowship grant. Infrastructure provided by UGC, India under UPE and CPEPA schemes and Department of Biotechnology, India under DISC facility is highly acknowledged. The funding source had no role in study design; collection, analysis and interpretation of data; in writing of report and in decision to submit the article for publication.

\section{Authorship Contributions}

Experiments were designed and conceived by G.K., T.K., S.M., V.S. and S.S.L. Experiments were performed by T.K., S.M. and V.S. Data was analyzed by G.K., T.K., S.M., V.S. and S.S.L. Lab facilities/ reagents/materials/analysis tools provided and contributed by G.K. Manuscript was written by G.K., T.K., S.M. and S.S.L.

\section{References}

1 Binder DK, Steinhäuser C: Functional changes in astroglial cells in epilepsy. Glia 2006;54: 358-368.

2 Friedman A, Kaufer D, Heinemann U: Bloodbrain barrier breakdown-inducing astrocytic transformation: novel targets for the prevention of epilepsy. Epilepsy Res 2009;85:142-149.

3 Rogawski MA, Löscher W: The neurobiology of antiepileptic drugs. Nat Rev Neurosci 2004; 5:553-564.

4 Perucca P, Gilliam FG: Adverse effects of antiepileptic drugs. Lancet Neurol 2012;11:792802.

5 Kudin AP, Debska-Vielhaber G, Vielhaber S, et al: The mechanism of neuroprotection by topiramate in an animal model of epilepsy. Epilepsia 2004;45:1478-1487.

6 Willmore LJ: Antiepileptic drugs and neuroprotection: current status and future roles. Epilepsy Behav 2005;7(suppl 3):S25-S28.

7 Rouaux C, Panteleeva I, René F, Gonzalez de Aguilar JL, et al: Sodium valproate exerts neuroprotective effects in vivo through CREBbinding protein-dependent mechanisms but does not improve survival in an amyotrophic lateral sclerosis mouse model. J Neurosci 2007;27:5535-5545.

8 Bruno V, Sortino MA, Scapagnini U, et al: Antidegenerative effects of $\mathrm{Mg}(2+)$-valproate in cultured cerebellar neurons. Funct Neurol 1994; 10:121-130.

9 Scheinfeld N: The role of gabapentin in treating diseases with cutaneous manifestations and pain. Int J Dermatol 2003;42:491-495.

10 Magnus L: Nonepileptic uses of gabapentin. Epilepsia 1999;40(suppl 6):S66-S72.

11 Baydas G, Sonkaya E, Tuzcu M, et al: Novel role for gabapentin in neuroprotection of central nervous system in streptozotocine-induced diabetic rats. Acta Pharmacol Sin 2005; 26:417-422.

12 Li H, Graber KD, Jin S, et al: Gabapentin decreases epileptiform discharges in a chronic model of neocortical trauma. Neurobiol Dis 2012;48:429-438.

13 Kale A, Börcek AÖ, Emmez H, et al: Neuroprotective effects of gabapentin on spinal cord ischemia-reperfusion injury in rabbits. J Neurosurg Spine 2011;15:228-237.

14 Hansen MB, Nielsen SE, Berg K: Re-examination and further development of a precise and rapid dye method for measuring cell growth/ cell kill. J Immunol Methods 1989;119:203210.

15 Mishra R, Kaur G: Aqueous ethanolic extract of Tinospora cordifolia as a potential candidate for differentiation based therapy of glioblastomas. PLoS One 2013;8:e78764.
16 Boss BD, Gozes I, Cowan WM: The survival of dentate gyrus neurons in dissociated culture. Brain Res 1987;433:199-218.

17 Ma B, Xiang Y, Li T, et al: Inhibitory effect of topiramate on Lewis lung carcinoma metastasis and its relation with AQP1 water channel. Acta Pharmacol Sin 2004;25:54-60.

18 Ma B, Pan Y, Song Q, et al: The effect of topiramate on tumor-related angiogenesis and on the serum proteome of mice bearing Lewis lung carcinoma. Eur J Pharmacol 2011;663: 9-16.

19 Wang TH, Wang HS, Soong YK: Paclitaxelinduced cell death: where the cell cycle and apoptosis come together. Cancer 2000;88: 2619-2628.

20 Gaul L, Mandl-Weber S, Baumann P, et al: Bendamustine induces G2-arrest by ATMCHK2-P53. Haematologica 2007;92:139.

21 Catalano MG, Fortunati N, Pugliese M, et al: Valproic acid induces apoptosis and cell cycle arrest in poorly differentiated thyroid cancer cells. J Clin Endocrinol Metab 2005;90:13831389.

22 Sofroniew MV: Molecular dissection of reactive astrogliosis and glial scar formation. Trends Neurosci 2009;32:638-647.

23 Pekny M, Nilsson M: Astrocyte activation and reactive gliosis. Glia 2005;50:427-434. 
24 Rossi AR, Angelo MF, Villarreal A, et al: Gabapentin administration reduces reactive gliosis and neurodegeneration after pilocarpineinduced status epilepticus. PLoS One 2013; 8:e78516.

25 Heikkila JJ: Heat shock gene expression and development. II. An overview of mammalian and avian developmental systems. Dev Genet 1993;14:87-91.

26 Loones MT, Chang Y, Morange M: The distribution of heat shock proteins in the nervous system of the unstressed mouse embryo suggests a role in neuronal and non-neuronal differentiation. Cell Stress Chaperones 2000; 5:291-305.

27 Nylandsted J, Brand K, Jäättelä M: Heat shock protein 70 is required for the survival of cancer cells. Ann N Y Acad Sci 2000;926:122125.

28 Yin GZ, Tu KS, Han SS, et al: [Downregulation of HSP70 gene expression and apoptosis in human hepatocellular carcinoma SMMC7721 cells induced by nimesulide in vitro]. $\mathrm{Xi}$ Bao Yu Fen Zi Mian Yi Xue Za Zhi 2012;28: 933-936.

29 Feng Z, Porter AG: NF-kappaB/Rel proteins are required for neuronal differentiation of SH-SY5Y neuroblastoma cells. J Biol Chem 1999;274:30341-30344.
30 Peng GS, Li G, Tzeng NS, et al: Valproate pretreatment protects dopaminergic neurons from LPS-induced neurotoxicity in rat primary midbrain cultures: role of microglia. Mol Brain Res 2005;134:162-169.

31 Penas C, Verdú E, Asensio-Pinilla E, et al: Valproate reduces $\mathrm{CHOP}$ levels and preserves oligodendrocytes and axons after spinal cord injury. Neuroscience 2011;178:33-44.

32 Brambilla P, Perez J, Barale F, et al: GABAergic dysfunction in mood disorders. Mol Psychiatr 2003;8:721-737, 715.

33 Emrich HM, von Zerssen D, Kissling W, et al: Effect of sodium valproate on mania. The GABA-hypothesis of affective disorders. Arch Psychiatr Nervenkr (1970) 1980;229:116.

34 Cox ET, Brennaman LH, Gable KL, et al: Developmental regulation of neural cell adhesion molecule in human prefrontal cortex. Neuroscience 2009;162:96-105.

35 Chen ZP, Schell JB, Ho CT, et al: Green tea epigallocatechin gallate shows a pronounced growth inhibitory effect on cancerous cells but not on their normal counterparts. Cancer Lett 1998;129:173-179.

36 Rusnak DW, Lackey K, Affleck K, et al: The effects of the novel, reversible epidermal growth factor receptor/ErbB-2 tyrosine kinase inhibitor, GW2016, on the growth of human normal and tumor-derived cell lines in vitro and in vivo. Mol Cancer Ther 2001;1: 85-94.
37 Beecken WD, Engl T, Ogbomo H, et al: Valproic acid modulates NCAM polysialylation and polysialyltransferase mRNA expression in human tumor cells. Int Immunopharmacol 2005;5:757-769.

38 Kiss JZ, Rougon G: Cell biology of polysialic acid. Curr Opin Neurobiol 1997;7:640-646.

39 Parkash J, Kaur G: Potential of PSA-NCAM in neuron-glial plasticity in the adult hypothalamus: role of noradrenergic and GABAergic neurotransmitters. Brain Res Bull 2007;74:317-328.

40 Kumar S, Parkash J, Kataria H, et al: Enzymatic removal of polysialic acid from neural cell adhesion molecule interrupts gonadotropin releasing hormone $(\mathrm{GnRH})$ neuron-glial remodeling. Mol Cell Endocrinol 2012;348: 95-103.

41 Lote K, Stenwig AE, Skullerud K, Hirschberg $\mathrm{H}$ : Prevalence and prognostic significance of epilepsy in patients with gliomas. Eur J Cancer 1998;34:98-102.

42 Beaumont A, Whittle IR: The pathogenesis of tumour associated epilepsy. Acta Neurochir (Wien) 2000;142:1-15

43 Tandon PN, Mahapatra AK, Khosla A: Epileptic seizures in supratentorial gliomas. Neurol India 2001;49:55-59. 